\title{
Erratum: Microstructure identification via detrended fluctuation analysis of ultrasound signals [Phys. Rev. E 87, 043304 (2013)]
}

Paulo G. Normando, Romão S. Nascimento, Elineudo P. Moura, and André P. Vieira (Received 19 October 2016; published 3 November 2016)

DOI: 10.1103/PhysRevE.94.059903

The original published version of the paper contains two mistakes regarding the values of the damping factor and of the transducer length listed on page 2. The correct value of the damping factor is $\gamma=1293.1 \mathrm{~m}^{-1}$ and the correct value of the transducer length is $2.32 \mathrm{~mm}$. The remaining values are correct and the mistakes corrected here do not affect any of the reported results. 